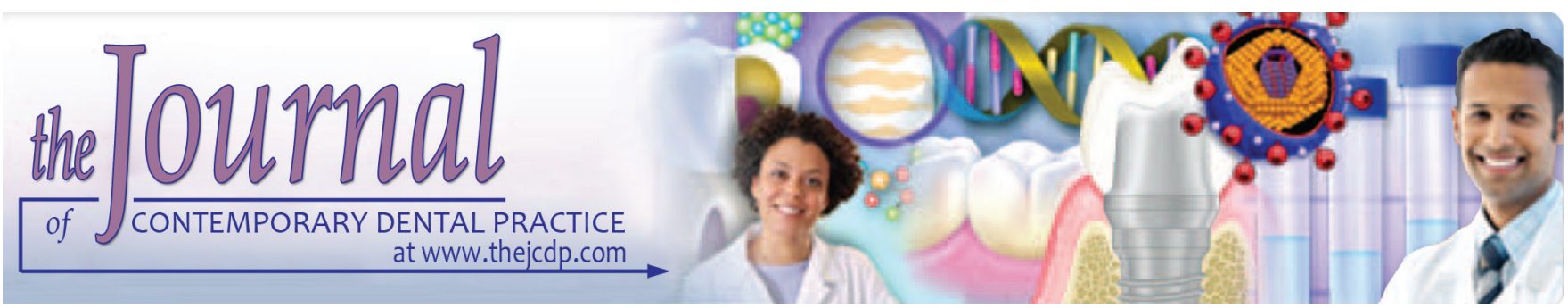

\title{
Remineralization Potential of Three Different Dentifrices using Raman Spectroscopy and Confocal Laser Scanning Microscope
}

${ }^{1}$ Tisson V Job, ${ }^{2}$ Girish T Narayana, ${ }^{3}$ Kishan K Venkappa, ${ }^{4}$ K Binu Nathan, ${ }^{5}$ Shameem Ahsan, ${ }^{6}$ Jayakkodi Harikaran

\section{ABSTRACT}

Aim: The aim of this study was to compare the remineralization potential of three different dentifrices using Raman spectroscopy and confocal laser scanning microscopy (CLSM).

Materials and methods: Totally, 30 extracted intact impacted third molar teeth were selected and the crown of each tooth in a group was separated from the root and longitudinally sectioned into four parts with each section under a subgroup, of which one section was an untreated section, the second and the third sections were demineralized in a demineralizing solution, and the third section was remineralized after demineralization. The teeth in the three groups were demineralized for 4 days and then treated with $0.21 \%$ sodium fluoride dentifrice with tricalcium phosphate, casein phosphopeptide-amorphous calcium phosphate (CPP-ACP), and NovaMin for 14 days, following which the teeth surfaces were studied using Raman spectroscopy and CLSM to assess the remineralization potential of the three dentifrices. The data were recorded and analyzed statistically.

Results: Raman spectroscopic analysis revealed better remineralization with CPP-ACP, which was statistically significant from the groups treated with the NovaMin dentifrice and the fluoridecontaining dentifrice.Confocal laser scanning microscopic examination also revealed significant differences between the three groups with the NovaMin-containing dentifrice demonstrating a greater remineralization of the surface when compared with the CPP-ACP dentifrice. The teeth samples treated with

\footnotetext{
1,4-6Department of Conservative Dentistry and Endodontics Educare Institute of Dental Sciences, Malappuram, Kerala, India

${ }^{2}$ Department of Conservative Dentistry and Endodontics, Coorg Institute of Dental Sciences, Virajpet, Karnataka, India

${ }^{3}$ Department of Conservative Dentistry and Endodontics, K M Shah Dental College \& Hospital, Vadodara, Gujarat, India

Corresponding Author: Tisson V Job, Department of Conservative Dentistry and Endodontics, Educare Institute of Dental Sciences, Malappuram, Kerala, India, e-mail: tissonisnow@gmail.com
}

fluoride-containing dentifrice demonstrated the least remineralization among the three groups.

Conclusion: It can be concluded that the demineralized samples of teeth treated with CPP-ACP showed the highest concentration of phosphate ions when analyzed using Raman spectroscopy, and the microscopic examination using confocal laser revealed a better surface remineralization of the demineralized samples when treated with the NovaMin technology.

Clinical significance: There is a great need to find ways to enhance the remineralization process and transfer such knowledge into clinical therapy to alter caries balance for the better, especially in individuals with a high cariogenic bacterial challenge.

Keywords: Casein phosphopeptide-amorphous calcium phosphate, Fluoride, NovaMin, Remineralization, Tricalcium phosphate.

How to cite this article: Job TV, Narayana GT, Venkappa KK, Nathan KB, Ahsan S, Harikaran J. Remineralization Potential of Three Different Dentifrices using Raman Spectroscopy and Confocal Laser Scanning Microscope. J Contemp Dent Pract 2018;19(4):420-425.

Source of support: Nil

Conflict of interest: None

\section{INTRODUCTION}

Dental caries is initiated through the demineralization of tooth hard tissues, which is a "pH-driven phenomenon". This occurs by organic acids produced from fermentable carbohydrates by dental plaque cariogenic bacteria. Demineralization and remineralization can be considered as a dynamic process, characterized by the flow of calcium and phosphate out of and back into the tooth enamel, which should be balanced to prevent the progression of caries. ${ }^{1,2}$

White spot lesions are the earliest macroscopic evidence of enamel caries. The majority of demineralization 
in white-spot lesions occurs in the subsurface region of enamel. This subsurface demineralization increases porosity and changes the optical properties of enamel. Typically, the enamel surface layer stays intact during subsurface demineralization, but without treatment will eventually collapse into a full cavity. ${ }^{3}$

Remineralization is the natural repair process for caries lesions. This occurs when calcium and phosphate in water among enamel or dentin crystals recrystallize on the surface of existing crystal remnants. Remineralization requires saliva or some other liquid to facilitate the transport of calcium and phosphate ions into the tooth. ${ }^{4}$

There are increasing numbers of promising technologies aimed at enhancing tooth mineralization or preventing tooth decay, yet relatively few technologies are brought to market with substantiated claims of anticariogenic performances, even when favorable clinical performances have been established. ${ }^{5}$

Fluoride-containing dentifrices have significantly declined caries experience. The efficacy of these dentifrices has been attributed to their ability to incorporate fluoride ions into plaque and enamel. ${ }^{6}$ The CPP-ACP technology stabilizes high concentrations of calcium and phosphate ions at the tooth surface binding to pellicle and plaque, which provides a highly effective means for elevating calcium levels in dental plaque fluid, something which is desirable for enhancing remineralization. The NovaMin technology is based on calcium sodium phosphosilicate bioactive glass, which is claimed to release calcium and phosphate ions intraorally to help the selfrepair process of teeth. ${ }^{7}$

Raman scattering spectroscopy is considered as one of the useful techniques for assessing quantitatively the levels of demineralization and remineralization. This method allows the characterization, analysis of the concentration of ions, like carbonate, acid phosphate, as well as identifying other organic or mineral compounds. ${ }^{8}$

The imbibitions of a fluorescent dye into the porosities of demineralized enamel enable rapid quantitative analysis of thick samples (half a tooth) using CLSM. The mean gray values are plotted within the lesion to determine the fluorescence levels. The greater fluorescence corresponds to higher gray values (thus, smaller values are indicative of less porosity and dye penetration, or more mineral). ${ }^{9}$

Hence, this study was carried out to compare the remineralization potential of three different dentifrices using Raman's spectroscopy and CLSM.

\section{MATERIALS AND METHODS}

This in vitro study was conducted in the Department of Conservative Dentistry and Endodontics, Coorg Institute of Dental Sciences, Virajpet, India, in collaboration with the Department of Raman and the Department of Confocal Laser, Consortium for Scientific Research, Indore, India.

Thirty extracted intact impacted third molar teeth were collected, cleaned of any debris, and stored in normal saline until further use. The teeth were preselected to exclude those with cracks or any external structural faults.

\section{Methods}

\section{Dentifrice Supernatants}

The dentifrice supernatants were prepared by thoroughly mixing a 3:1 ratio of distilled water and dentifrice (by weight) and then centrifuging at 4,000 rpm for 20 minutes at $28^{\circ} \mathrm{C}$. The $\mathrm{pH}$ of the dentifrice supernatants was recorded. The fluoride-containing dentifrice supernatant solution had a $\mathrm{pH}$ of 7.8, CPP-ACP supernatant solution had a $\mathrm{pH}$ of 8.6 , and the NovaMin-containing dentifrice supernatant recorded a $\mathrm{pH}$ of 9.4 .

\section{De-/Remineralizing Solution Preparation}

The buffered demineralizing and remineralizing solutions were made up of analytical-grade chemicals and distilled water. The demineralizing solution, which contained 2.2 $\mathrm{mM} \mathrm{CaCl}$, $2.2 \mathrm{mM} \mathrm{KH}_{2} \mathrm{PO}_{4}$, and $0.05 \mathrm{M}$ acetic acid, had the $\mathrm{pH}$ adjusted to 4.4 with $1 \mathrm{M} \mathrm{KOH}$. The remineralizing solution, which contained $1.5 \mathrm{mM} \mathrm{CaCl}_{2}, 0.9 \mathrm{mM}$ $\mathrm{NaH}_{2} \mathrm{PO}_{4}$, and $0.15 \mathrm{M} \mathrm{KCl}$, had a $\mathrm{pH}$ of 7.0.

\section{Preparation of Rhodamine B Solution}

A $0.1 \mathrm{mM}$ of rhodamine B solution was prepared by adding $23.95 \mathrm{mg}$ of rhodamine B dye to $500 \mathrm{~mL}$ of distilled water.

The teeth were randomly categorized into three groups with ten teeth in each group. The crown of each tooth in a group was separated from the root and longitudinally sectioned.

This gives 10 sections in each subgroup and a total of 30 sections in each group. The total sample size for the study was 90 .

\section{Grouping of Teeth}

- Group I: Teeth treated with supernatant solution of CLINPRO [0.21\% sodium fluoride with tricalcium phosphate TCP)].

- Group Ia: The untreated section

- Group Ib: The demineralized section (Fig. 1)

- Group Ic: The section remineralized with CLINPRO after demineralization (Fig. 2).

- Group II: Teeth treated with supernatant solution of Tooth Mousse (CPP-ACP)

- Group IIa: The untreated section 


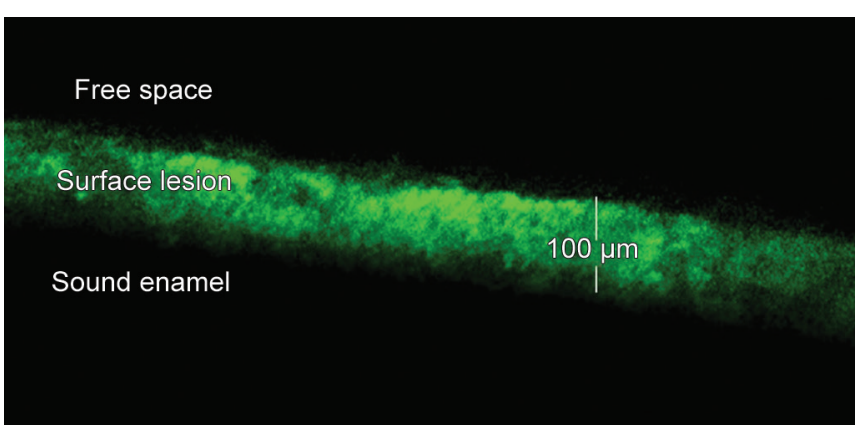

Fig. 1: The CLSM image of demineralized section of tooth (group I)

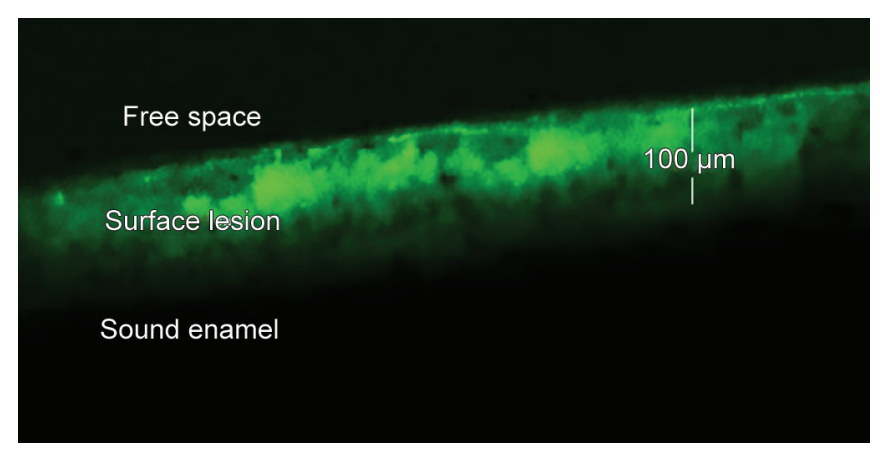

Fig. 3: The CLSM image of demineralized section of tooth (group II)

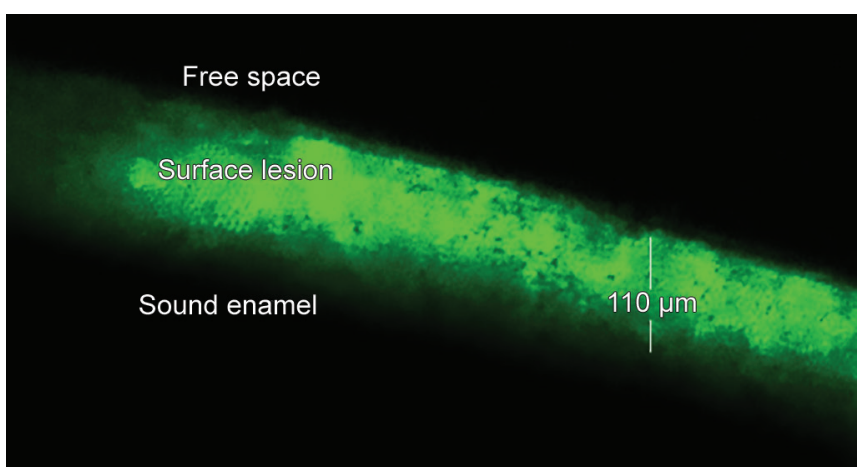

Fig. 5: The CLSM image of demineralized section of tooth (group III)

- Group IIb: The demineralized section (Fig. 3)

- Group IIc: The section remineralized with Tooth Mousse after demineralization (Fig. 4).

- Group III: Teeth treated with supernatant solution of Nupro Nusolutions (neutral fluoride toothpaste with calcium sodium phosphosilicate).

- Group IIIa: The untreated section

- Group IIIb: The demineralized section (Fig. 5)

- Group IIIc: The section remineralized with Nupro Nusolutions after demineralization (Fig. 6).

\section{Lesion Formation}

Three of the four quadrant sections of each tooth sample were selected for the study. Two of the sections of each tooth sample were demineralized in the demineralizing

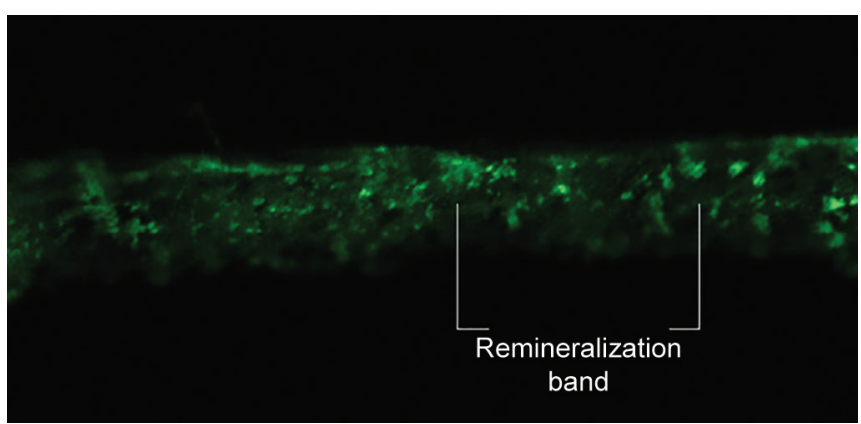

Fig. 2: The CLSM image of tooth section remineralized with $0.21 \%$ sodium fluoride with tricalcium phosphate (group I)

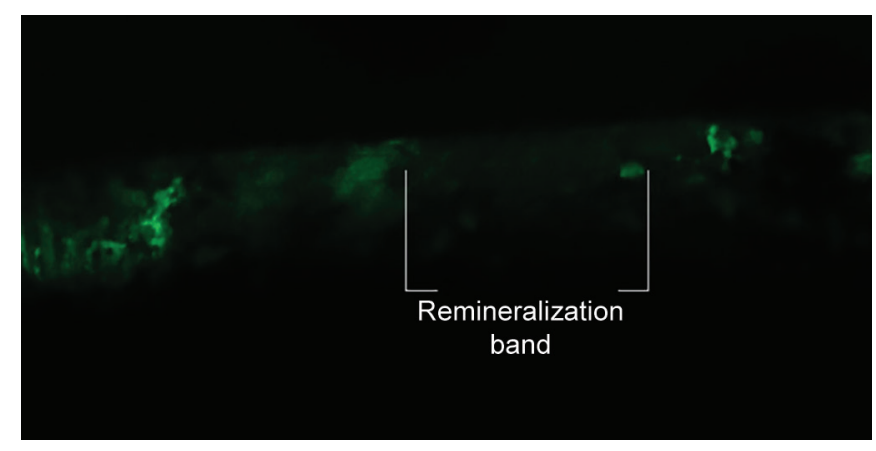

Fig. 4: The CLSM image of tooth section remineralized with CPP-ACP (group II)

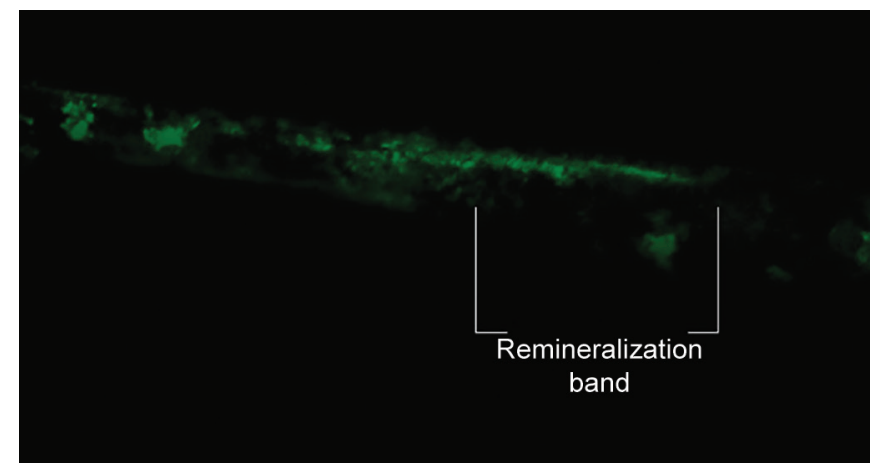

Fig. 6: The CLSM image of tooth section remineralized with NovaMin (group III)

solution for 96 hours, so that 90 to $120 \mu \mathrm{m}$ deep lesions were formed. One section of the tooth was stored in distilled water, and the other section was subjected to $\mathrm{pH}$ cycling.

\section{pH Cycling Model}

All of the specimens to be treated with each dentifrice (the third section of each tooth) underwent $\mathrm{pH}$ cycling in the following manner:

- The sections were treated with the respective dentifrice supernatant ( $5 \mathrm{~mL}$ per section) for 60 seconds and then rinsed thoroughly with distilled water

- Each section was immersed in the demineralizing solution (10 $\mathrm{mL}$ per section) for 3 hours followed by thorough rinsing with distilled water 
- Sections were immersed in remineralizing solution for 2 hours and then rinsed in distilled water

- A 60 seconds treatment with the selected dentifrice supernatant $(5 \mathrm{~mL}$ per section) again before and after the second demineralizing for 3 hours

- Sections were then placed in the remineralizing solution overnight.

The $\mathrm{pH}$ cycling went on for 14 days.

All solutions (demineralizing, remineralizing, and dentifrice supernatants) were freshly prepared for each cycle; separate containers were used for each group throughout the experimental period. The $\mathrm{pH}$ of the demineralizing and remineralizing solutions was measured before each cycle.

\section{Methodology for Raman Spectroscopy}

Quantitative evaluation was done using Raman spectroscopy. The spectra were recorded with Jobin Yvon Horibra LabRAM HR. The LabRAM HR systems provide high spectroscopic resolution and a unique wavelength range capability that offers both great flexibility and high performance. The laser source is an argon laser with a $488 \mathrm{~nm}$ wavelength.

The output power was fixed to $100 \mathrm{mw}$ so that the surface would not be overheated. The laser beam was focused onto the sample surface using the light-optical microscope of the micro-Raman spectroscope. The focal size was estimated to be $10 \mu \mathrm{m}$ in diameter. Moreover, a comparison is drawn between the three groups to assess the remineralizing potential of each dentifrice.

\section{Methodology for CLSM}

The sections under the subgroups of groups I, II, and III are then stained with $0.1 \mathrm{mM}$ rhodamine B solution for 24 hours and then cross-sectioned and viewed under the confocal microscope for analyzing the demineralization and remineralization.

The stained sections were fixed and mounted on the microscope glass slide using modeling wax. Using a $\times 10$ objective, the specimens were illuminated with an argon laser at 50\% intensity using a $488 \mathrm{~nm}$ excitation wavelength. Confocal slits were set at $25 \mu \mathrm{m}$ with a 515 $\mathrm{nm}$ long-pass filter. Areas were scanned planoparellel to the cut surface of the specimen.

Sound enamel (untreated specimen) registers nearzero fluorescence (gray scale value $\sim 0$ ) and appears pitch black. Lesions (demineralized specimen) slightly autofluorescence, but the imbibition of the rhodamine dye $(0.1 \mathrm{mM})$ allows the porous demineralized layer to fill and appear with considerable contrast (higher gray values). Remineralized samples showed a reduced fluorescence (smaller gray values, which are indicative of less porosity and dye penetration or more mineral).
The reduction in the fluorescence (gray values) was compared between the three groups to assess the remineralization potential of each dentifrice.

\section{RESULTS}

Group I showed a decrease of phosphate content by $19.27 \%$, group II by $19.75 \%$, and group III by $19.37 \%$. One-way analysis of variance (ANOVA) revealed nonsignificant difference $(p>0.05)$ between $\%$ decrease of values after demineralization between the three groups (Table 1 ).

In Table 2, group I showed an increase of phosphate content by $10.46 \%$, group II $14.02 \%$, and group III $11.19 \%$. One-way ANOVA revealed a significant difference $(p<0.05)$ between $\%$ increases of values from demineralization to remineralization between the three groups. Post hoc analysis (Table 3) revealed a significant difference between groups I and II $(\mathrm{p}<0.05)$ and between groups II

Table 1: The intensity of shift of concentration of phosphate ions and comparison of the amount of demineralization between the three groups

\begin{tabular}{lllll}
\hline Groups & $n$ & Mean $\pm S D$ & $f$-value & $p$-value \\
\hline $\begin{array}{l}\text { Group I (0.21\% } \\
\text { sodium fluoride with }\end{array}$ & 10 & $19.2797 \pm 1.06521$ & 1.498 & 0.242 \\
$\begin{array}{l}\text { TCP) } \\
\text { Group II (CPP-ACP) }\end{array}$ & 10 & $19.7526 \pm 0.23352$ & & \\
$\begin{array}{l}\text { Group III (neutral } \\
\text { sodium fluoride with } \\
\text { calcium sodium }\end{array}$ & 10 & $19.3741 \pm 0.27326$ & & \\
phosphosilicate) & & & & \\
\hline
\end{tabular}

SD: Standard deviation

Table 2: The intensity of the shift of concentration of phosphate ions and comparison of the amount of remineralization between the three groups

\begin{tabular}{llllll}
\hline Groups & $n$ & Mean & $S D$ & $f$-value & $p$-value \\
\hline $\begin{array}{l}\text { Group I (0.21\% } \\
\text { sodium fluoride } \\
\text { with TCP) }\end{array}$ & 10 & 10.4572 & 0.84731 & 22.239 & $0.000^{*}$ \\
$\begin{array}{l}\text { Group II (CPP- } \\
\text { ACP) }\end{array}$ & 10 & 14.0250 & 1.99871 & & \\
$\begin{array}{l}\text { Group III } \\
\text { (neutral sodium } \\
\text { fluoride with } \\
\text { calcium sodium } \\
\text { phosphosilicate) }\end{array}$ & 10 & 11.1999 & 0.25970 & & \\
\hline $\begin{array}{l}\text { SD: Standard deviation; *Statistically significant } \\
\end{array}$ & & & & & \\
\end{tabular}

Table 3: Intergroup comparison of the amount of remineralization (post hoc analysis)

\begin{tabular}{lllll}
\hline & & $\begin{array}{l}\text { Mean } \\
\text { Groups comparison }\end{array}$ & $\begin{array}{l}\text { Standard } \\
\text { difference }\end{array}$ & error \\
\hline Group I & Group II & -3.56778 & 0.56452 & $0^{*}$ \\
& Group III & -0.74271 & 0.56452 & 0.399 \\
\multirow{2}{*}{ Group II } & Group I & 3.56778 & 0.56452 & $0^{*}$ \\
& Group III & 2.82507 & 0.56452 & $0^{*}$ \\
\hline
\end{tabular}

*Significant 
Table 4: The intensity of shift of the concentration of phosphate ions after remineralization compared with the untreated samples (paired t-test)

\begin{tabular}{lllllll}
\hline & & \multicolumn{2}{l}{ Paired } & & & \\
\cline { 3 - 3 } & & Mean & & Df & Sig. (2-tailed) \\
\hline Group I & U1-R1 & 3262.2000 & 16.657 & 9 & $0^{*}$ \\
Group II & U2-R2 & 2489.6000 & 15.397 & 9 & $0^{*}$ \\
Group III & U3-R3 & 3063.6000 & 146.387 & 9 & $0^{*}$
\end{tabular}

U: Untreated sample; R: Remineralized sample; *Significant;

Df: Degree of freedom

Table 6: Intergroup comparison of the percentage of decrease in fluorescence following remineralization (post hoc analysis)

\begin{tabular}{lllll}
\hline & & Mean & Standard & \\
Groups comparison & difference & error & $p$-value \\
\hline Group I & Group II & -11.57816 & 2.55276 & $0^{*}$ \\
\multirow{4}{*}{ Group II } & Group III & -18.97851 & 2.55276 & $0^{*}$ \\
& Group I & 11.57816 & 2.55276 & $0^{*}$ \\
& Group III & -7.40036 & 2.55276 & $0.019^{*}$ \\
\hline
\end{tabular}

*Significant

and III $(\mathrm{p}<0.05)$. No significant difference was found between groups I and III ( $p>0.05)$.

In Table 4, paired t-tests revealed that the differences between the intensity of phosphate ions in the untreated and the remineralized samples in all the three groups were statistically significant $(\mathrm{p}<0.05)$.

Group I showed an increase of remineralization (reduction in fluorescence) by $73.48 \%$, group II by $85.06 \%$, and group III by $92.46 \%$. Statistical analysis using one-way ANOVA revealed a significant difference $(p<0.05)$ in the percentage of remineralization among the three groups (Table 5). Post hoc analysis (Table 6) revealed significant differences between groups I and II $(p<0.05)$, between groups I and III $(\mathrm{p}<0.05)$, and between groups II and III $(\mathrm{p}<0.05)$

\section{DISCUSSION}

Crystals at the tooth surface regularly go through natural periods of mineral loss (demineralization) and mineral gain (remineralization), particularly surfaces covered by undisturbed (stagnant) films, i.e., dental plaque.

Several techniques have been developed to measure mineral changes on human enamel. These techniques include microhardness measurement of enamel surface, microhardness measurement of enamel cross-sections, polarized light microscopy, different microradiography techniques, iodine absorptiometry, and light scattering. All methods have their share of advantages and disadvantages. ${ }^{10}$

Only a few studies have assessed the remineralization of teeth using Raman spectroscopy and CLSM. ${ }^{3,8,9}$

Taking into consideration the excellent remineralization capabilities of these dentifrices, this study was aimed at comparing the remineralization potential of the same using Raman spectroscopy and CLSM.
Table 5: Intergroup comparison of the percentage of decrease in fluorescence following remineralization (ANOVA)

\begin{tabular}{lllll}
\hline Groups & $n$ & Mean $\pm S D$ & $f$-value & $p$-value \\
\hline $\begin{array}{l}\text { Group I (0.21\% } \\
\text { sodium fluoride with }\end{array}$ & 10 & $73.4844 \pm 7.54979$ & 28.082 & $0.000^{*}$ \\
TCP) & & & & \\
$\begin{array}{l}\text { Group II (CPP-ACP) } \\
\text { Group III (neutral }\end{array}$ & 10 & $85.0626 \pm 5.66656$ & & \\
$\begin{array}{l}\text { sodium fluoride with } \\
\text { calcium sodium }\end{array}$ & & $92.4629 \pm 2.93936$ & & \\
phosphosilicate) & & & & \\
\hline
\end{tabular}

SD: Standard deviation; *Statistically significant

In the present study, group II (CPP-ACP) shows the increase of phosphate content by $14.02 \%$. These observations are inconsistent with recent studies by Reynolds et $\mathrm{al}^{11}$ and Rose, ${ }^{12,13}$ in which they accounted for the enhanced levels of calcium and phosphate by the use of a CPP-ACP dentifrice/mouth rinse. They also reported an increased level of calcium and inorganic phosphate ions in supragingival plaque, with the CPP bound to the plaque matrix and onto the surface of bacteria.

The ability of the CPP-ACP technology (group II) to stabilize high concentrations of calcium and phosphate at the tooth surface by binding to pellicle and plaque has accounted for the highest concentration of phosphate ions $(14.02 \%)$, its superior remineralizing effect, and also its closest value to the corresponding untreated specimens. ${ }^{7}$

The enhanced intensity in the peak of the Raman band following treatment with $\mathrm{CPP}-\mathrm{ACP}$ corresponds to more hydroxyapatite formation, which explains the enhanced remineralization, as solution containing the same would drive mineral back into the tooth. ${ }^{8}$

The ability of NovaMin to release sodium, calcium, and phosphate ions when the dentifrice interacts with the aqueous environment it is present in has resulted in the formation of a crystalline hydroxylcarbonate apatite layer that is structurally and chemically similar to natural tooth mineral (group III), which may have resulted in a high concentration of phosphate ions $(11.19 \%)$ on the tooth surface. ${ }^{3}$

The sentence meant that the samples in the group showed the next closest value to Group II i.e comparing the phosphate ions. These findings are consistent with a recent scanning electron microscopy and microhardness study by Burwell et $\mathrm{al}^{3}{ }^{3}$ which demonstrated that NovaMin alone, and in combination of fluoride, can enhance the remineralization of enamel and dentin lesions, as well as prevent demineralization from acidic challenges.

The fluoride-containing dentifrice (group I) due to the presence of lower concentrations of TCP may have resulted in a reduced shift for the intensity of phosphate ions $(10.45 \%)$ when compared with the CPP-ACP technology. The use of the dentifrice may have formed calcium-phosphate complexes, which have hindered the 
remineralization process. Fluoride present in the dentifrice can form calcium fluoride, which may have inhibited remineralization by lowering the levels of bioavailable calcium and fluoride. ${ }^{7,14}$

The imbibition of a fluorescent dye into the porosities of demineralized enamel enables rapid quantitative analysis of thick samples (half a tooth) using CLSM.

When using CLSM for the evaluation of the depth of surface remineralization, the demineralized samples treated with the NovaMin technology (group III) showed a greater reduction in the fluorescence (smaller gray values) and clearer remineralization bands. The percentage of remineralization attained is $92.46 \%$.

The demineralized samples treated with CPP-ACP (group II) showed an increase in the depth of remineralization by $85.06 \%$ followed by the samples treated with $0.21 \%$ sodium fluoride dentifrice with TCP (group I), which accounted for a remineralization percentage of $73.48 \%$.

These results are similar to a study of Alaudin and Fontana ${ }^{15}$ using confocal laser scanning microscope for analysis for analysis has reported superior remineralizing abilities of a NovaMin containing dentifrice when compared with a fluoride-containing dentifrice, which is similar to the results obtained in this study.

It has been reported that the ability of NovaMin to provide calcium and phosphate to an eroded tooth surface may decrease mineral loss as well as remineralization to occur in surface defects. The release of these ions from NovaMin and the high $\mathrm{pH}$ of the dentifrice of 9.6 may have provided a solubility gradient in favor of mineral deposition. $^{14}$

Taking into account the few limitations of this study (organic components, such as oral bacteria or plaque were not considered), the demineralized samples of teeth treated with CPP-ACP (group II) showed the highest concentration of phosphate ions when compared with teeth samples treated with NovaMin and fluoride with TCP, when analyzed using Raman spectroscopy. No significant difference was observed between the NovaMin dentifrice and the fluoride-containing dentifrice.

The microscopic examination using confocal laser revealed a better surface remineralization of the demineralized samples when treated with NovaMin technology when compared with the samples treated with CPP-ACP dentifrice and the fluoride dentifrice with TCP.

The comparison of the different remineralizing technologies has brought in promising results. Further investigations may be required to validate the effectiveness of these agents.

\section{CONCLUSION}

It can be concluded that the demineralized samples of teeth treated with CPP-ACP showed the highest concentration of phosphate ions when compared with teeth samples treated with NovaMin and fluoride with TCP when analyzed using Raman spectroscopy.

The microscopic examination using confocal laser revealed a better surface remineralization of the demineralized samples when treated with the NovaMin technology when compared with the samples treated with CPP-ACP dentifrice and the fluoride dentifrice with TCP.

\section{REFERENCES}

1. Reynolds EC. Casein phosphopeptide-amorphous calcium phosphate: the scientific evidence. Adv Dent Res 2009 Aug;21(1):25-29.

2. Torrado A, Valiente M, Zhang W, Li Y, Munoz C A. Remineralization potential of a new toothpaste formulation: an in vitro study. J Contemp Dent Pract 2004 Feb;5(1):18-30.

3. Burwell AK, Litkowski LJ, Greenspan DC. Calcium sodium phosphosilicate (NovaMin): remineralization potential. Adv Dent Res 2009 Jul;21(1):35-39.

4. Featherstone JD. Remineralization, the natural caries repair process-the need for new approaches. Adv Dent Res 2009 Jul;21(1):4-7.

5. Pfarrer AM, Karlinsey RL. Challenges of implementing new remineralization technologies. Adv Dent Res 2009 Jul;21(1):79-82.

6. Rana R, Itthagarun A, King NM. Effects of dentifrices on artificial caries like lesions: an in vitro $\mathrm{pH}$ cycling study. Int Dent J 2007 Aug;57(4):243-248.

7. Walsh LJ. Contemporary technologies for remineralization therapies: a review. Int Dent SA 2009 Jan;4:34-46.

8. Hassanein OE, El-Brolossy T. An investigation about the remineralization potential of bioactive glass on artificially carious enamel and dentin using Raman spectroscopy. Egypt J Solids 2006 Jun;29(1):69-80.

9. González-Cabezas C, Fontana M, Dunipace AJ, Li Y, Fischer GM, Proskin HM, Stookey GK. Measurement of enamel remineralization using microradiography and confocal microscopy. A correlational study. Caries Res 1998 Sep;32(5): 385-392.

10. Kumar VL, Itthagarun A, King NM. The effect of casein phosphopeptide-amorphous calcium phosphate on remineralization of artificial caries-like lesions: an in vitro study. Aust Dent J 2008 Mar;53(1):34-40.

11. Reynolds EC, Cai F, Shen P, Walker GD. Retention in plaque and remineralization of enamel lesions by various forms of calcium in a mouthrinse or sugar-free chewing gum. J Dent Res 2003 Mar;82(3):206-211.

12. Rose RK. Effects of an anticariogenic casein phosphopeptide on calcium diffusion in streptococcal model dental plaques. Arch Oral Biol 2000 Jul;45(7):569-575.

13. Rose RK. Binding characteristics of Streptococcus mutans for calcium and casein phosphopeptide. Caries Res 2000 Sep-Oct;34(5):427-431.

14. Yamasaki H, Litman A, Margolis HC. Effect of fluoride on artificial caries progression and repair in human enamel: regulation of mineral deposition and dissolution under in-vivo like conditions. Arch Oral Biol 2007 Feb;52(2):110-120.

15. Alaudin, SS.; Fontana, M. Evaluation of Nova Min as an adjunct to fluoride for caries lesion remineralization. NovaMin Research Reports. 2006. [cited 2009 Feb 26]. Available from: www.novamin.com. 